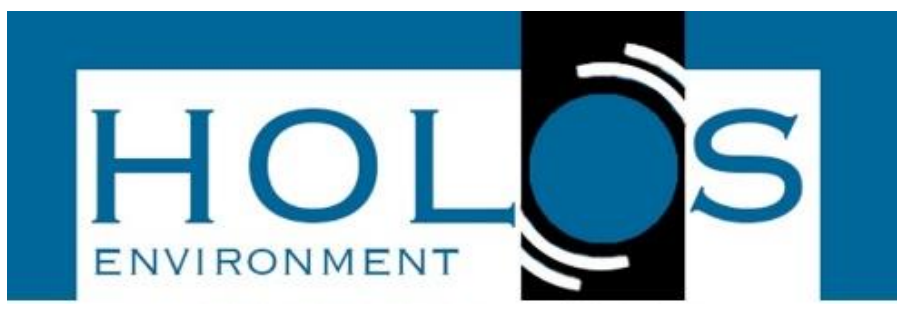

\title{
GRANULOMETRIA DO SOLO, PERCOLAÇÃO DA ÁGUA E TRANSPIRAÇÃO DA ÁREA VEGETADA NO CAMPUS DUSE RÜEGGER OMETTO - FHO - FUNDAÇÃO HERMÍNIO OMETTO, ARARAS-SP
}

\author{
GRANULOMETRY OF THE SOIL, WATER PERCOLATION AND \\ TRANSPIRATION OF THE VEGETATED AREA IN THE CAMPUS DUSE \\ RÜEGGER OMETTO - FHO - FUNDAÇÃO HERMÍNIO OMETTO, ARARAS-SP
}

\author{
Júlio Valentim Betioli ${ }^{1}$
}

Artigo recebido em: 30/08/2018 e aceito para publicação em: 17/06/2019.

DOI: http://dx.doi.org/10.14295/holos.v19i3.12308

\begin{abstract}
Resumo: O trabalho foi realizado no campus Duse Rüegger Ometto, da Fundação Hermínio Ometto em Araras, SP, com a finalidade de correlacionar a distribuição da granulometria do solo e sua permeabilidade com a capacidade de evapotranspiração da área vegetada, pelo método trabalhado de Biudes (2006) e sua relação com o microclima no local. Para coleta das amostras do solo foram utilizados uma cavadeira articulada e um termômetro a laser para medição da temperatura da amostragem no exato momento da coleta. Em cada ponto coletou-se três amostras com as profundidades variando entre 0-20 cm, 20-40 cm e 40-60 cm. Para a determinação da evapotranspiração as áreas foram obtidas por duas metodologias, a primeira feita in loco com trenas de 30 metros, e a segunda, delimitadas por satélites e calculadas com o software AutoCad. O solo, por meio das análises obtidas, está constituído por predominância de areia fina e média com pouca significância de argila. Isso indica um elevado índice de vazios e alta taxa efetiva de porosidade, o que favorece a percolação da água no solo. O método utilizado para calcular o potencial da transpiração em condições adotadas e descritas durante o experimento revelou o valor obtido de 34,7 m3.24horas-1 representa uma quantidade de água muito grande. Conclui-se que transpiração das plantas pode ser eficaz para manutenção do microclima e recomenda-se a inserção de vegetação na construção civil visto seus singulares benefícios e ambiente agradável.
\end{abstract}

Palavras-chave: Campus Universitário. Granulometria. Transpiração. Araras. Uniararas.

Abstract: The work was realized at the Duse Rüegger Ometto campus, from Herminio Ometto Foundation in Araras, SP, with the finality of correlate the granulometry distribution of the soil and its permeability with the capacity of evapotranspiration from the vegetated area, by the worked method of Biudes (2006) and its relation with the local microclimate. To the collect of the soil samples were used an articulated excavator and a laser thermometer to measure the temperature of the sampling in the moment of the collection. Three samples were collected in each point with depth varying between 0-20 $\mathrm{cm}, 20-40 \mathrm{~cm}$ and $40-60 \mathrm{~cm}$. To the determination of the evapotranspiration the areas were obtained by two methodologies, the first made in loco with 30 meters measuring tapes, and the second, delimited by satellites and calculated with the AutoCad software. The soil, through the analysis obtained, is constituted predominant by thin and medium sand with low significance of clay. The method to calculate the transpiration potential in conditions adopted and described during the experiment revealed the obtained valuo of $34,7 \mathrm{~m}^{3}$. 24 hours -1 represents a very large amount of water. It is concluded that plants transpiration can be effective to the microclimate maintainence and it is.

Keywords: University Campus. Granulometry. Transpiration. Araras. Uniararas.

${ }^{1}$ Fundação Hermínio Ometto (FHO), Araras, SP. E-mail: (juliobetioli@fho.br) 


\section{INTRODUÇÃO}

Nos dias atuais, a ocupação e o planejamento territorial urbano assumiu um papel que, em muitas situações, contradiz as leis físicas que regem essa dinâmica. Pois, nas áreas urbanas os Planos Diretores Municipais, de forma geral, orientaram ou permitiram edificar suas cidades se esquecendo da dinâmica entre o ambiente natural e as causas e efeitos da ocupação, principalmente quanto a impermeabilização do solo (SANTOS, RUFINO e BARROS FILHO, 2017).

Assim, a ecologia de paisagem e suas características tiveram que passar por grandes transformações, para satisfazer os anseios e necessidades antrópicas, como o crescimento e expansão urbana, levando a remoção da cobertura vegetal por desmatamento, erosão e remoção de grande quantidade de solo, subtração de grandes quantidades de recursos naturais, com impacto ambiental provocados pelas minerações, as monoculturas em largas escalas, exploração dos recursos hídricos para satisfazer as demandas e legislações que vigoravam em cada período e àquelas vigentes atualmente.

Nas cidades do interior de São Paulo as intervenções nos diferentes componentes da paisagem rural e urbana (relevo, solo, vegetação, clima e recursos hídricos) se intensificaram nas últimas décadas, devido ao crescente desenvolvimento urbano, industrial. Nesse cenário, ocorreu comprometimento da funcionalidade do sistema, interferindo no equilíbrio dinâmico. Levar em consideração a ocupação territorial urbana pode ser indicativo seguro a uma política de ocupação do espaço com a intenção de manter a integridades da ecologia da paisagem (LOSSARDO e DAINEZI, 2011).

Essa exploração desenfreada começou a indicar os reflexos da transformação antrópica a partir de dados climatológicos, tais como o de pluviosidade, das grandes secas em alguns períodos e em outros índices de chuvas em abundância, que podem também ser ocasionados de modo natural e cíclico, interferindo na fauna e flora nativos, que prejudica os serviços ecossistêmicos prestados por esses seres vivos e compartilhados como todos os outros grupos biológicos, em todas as teias alimentares, comprometimento da qualidade da água, do solo e do ar decorrentes de poluição de diferentes origens, surtos de doenças, como a dengue, a zica e shikungunya, são alguns exemplos de situações a que os moradores de grandes centros urbanos estão submetidos. 
A soma de tais fatores mostrou ao homem que sua existência se tornou insustentável, ou seja, satisfaz suas necessidades mais eminentes, tornando inacessíveis os recursos para que as próximas gerações. Nesse contexto surgem as ideias de sustentabilidade, green building, reaproveitamento e reciclagem, energias renováveis, diminuição no uso de combustíveis fósseis, reaproveitamento de água e desenvolvimento produtos e serviços que mitiguem o impacto do crescimento humano. Assim, os seres humanos estão percebendo a necessidade de incluir novos paradigmas relacionados ao meio ambiente, planejamento territorial e uso dos recursos naturais.

Tais conceitos estão ganhando espaço na sociedade de modo que desde a educação básica, as crianças aprendem a separar os materiais recicláveis e promover a economia de água e energia em casa e na escola. Nesse novo modelo, a reinserção de plantas no ambiente edificado e impermeabilizado é de incrível potencial para melhoria da qualidade de vida e até de apelo estético e arquitetônico (ABREU, 2008; LIMA JUNIOR, 2014; MARTINI e BIONDI, 2015).

A presença de vegetação remanescente de florestas nativas do local ou regenerada ou recuperada no meio urbano é essencial para estrutura e dinâmica da paisagem urbana, o que se denomina da Florestas Urbanas. Assim ocorrendo, o microclima se faz presente e melhora as condições ambientais onde há vegetação (ABREU, 2008; LIMA JUNIOR, 2014; MARTINI e BIONDI, 2015; TOLFFO, 2015). As plantas possuem um sistema vascular que operam a circulação de água e dos nutrientes que ela obtém do solo e dos processos fotossintéticos por meio dos estômatos elas transferem vapor de água desse sistema para a atmosfera, o que se denomina de evapotranspiração (LIMA JUNIOR, 2014). Este processo exerce também benefício ambiental, pois tem a capacidade de absorver calorias, gerando diminuição da temperatura do microclima local nas horas de maior calor (ABREU, 2008; MARTINI e BIONDI, 2015).

Segundo Nogueira (2006) e Amato-Lourenco et al (2016) as folhas, flores e até frutos são dotados de estômatos que são os canais de comunicação entre o sistema vascular e o ambiente externo a planta. Esse vapor de água liberado é extremamente benéfico à qualidade do ar, decantação de partículas de poeira e fuligem com consequente manutenção da qualidade de vida dos usuários de uma edificação. De acordo com as condições atmosféricas, a umidade do ar está baixa a trans- 
piração é intensificada, item primordial para considerar a transpiração como fator na melhoria da qualidade do ar inalado (Figura 1).

Dessa forma, as plantas contribuem para a manutenção do microclima, compensando, de certa maneira, o uso e ocupação do solo, decorrente da impermeabilização do solo, das construções que existem no local, da ausência de distribuição de árvores na área total, pavimentação excessiva e grande fluxo de veículos e pessoas estão contribuindo minimizar a retenção de calor e o aumento da umidade do ar, ocasionando uma situação ambiental confortável para aqueles que convivem nessas áreas (ABREU, 2008; GHENO et al., 2012; LIMA JUNIOR, 2014; MARTINI e BIONDI, 2015; AMATO-LOURENCO et al., 2016).

Figura 1 - Papeis ecossistêmicos realizados pelas plantas

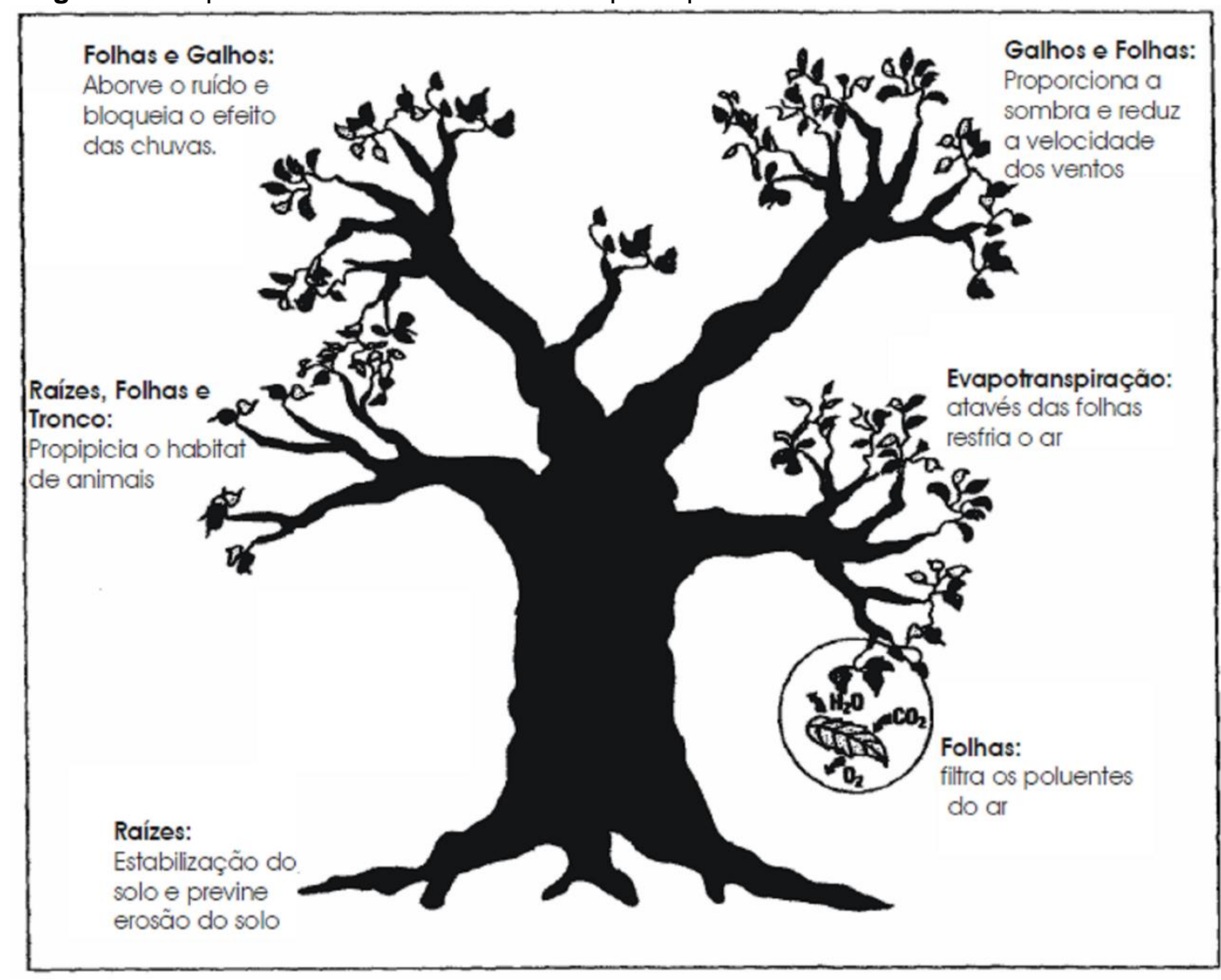

Fonte: Abreu (2008)

Neste cenário, um componente essencial que precisa ser levado em consideração é solo, pois sua influência pode contribuir de modo significativo nas condições do local amostrado. O solo pode ser constituído por partículas de tamanhos diversos, na serapilheira e nos diferentes horizontes, A a D. 
A granulometria do solo é uma das características mais estáveis e representa a distribuição quantitativa das partículas sólidas minerais quanto ao tamanho. É importante para a descrição, identificação e classificação do solo, com conotação quantitativa (FERREIRA, 2010).

Silva, Gonzalez e Silva Filho (2011) estudando no campus da UFPB quanto a área mais confortável do local, utilizando o índice de Thom que avalia o nível de conforto térmico, o tipo de cobertura de solo, o percentual de vegetação e de materiais permeáveis e impermeáveis, constataram que a área menos confortável compreendia um local cujo solo estava recoberto por areia e nas proximidades de uma rua asfaltada. Machado et al. (2013) obtiveram condições similares no campus da IFMT.

A permeabilidade está relacionada com o índice de vazios, logo com a sua porosidade. Quanto mais poroso for um solo, maior será seu índice de vazios, consequentemente mais permeável facilitando assim a infiltração.

A infiltração é o nome dado ao processo pelo qual a água atravessa a superfície do solo. É um processo de grande importância prática, pois afeta diretamente o escoamento superficial, que é o componente do ciclo hidrológico responsável pelos processos de erosão e inundações.

O conceito de porosidade é fator importante, pois pode interferir na aeração, na condução e na retenção de água nos diferentes horizontes, resistência à penetração e à ramificação das raízes o que dificulta a absorção de água e nutrientes disponíveis (RIBEIRO et al. 2007).

Portanto, analisar a granulometria e tipo de solo, que possibilita verificar a percolação na área escolhida, e a evapotranspiração da vegetação é fundamental, para conhecimento quanto a preservação e ampliação de espaços verdes numa IES, visando correlacionar os serviços ecossistêmicos prestados pela vegetação, principalmente quanto ao microclima, e por se tratar de um estudo inédito.

O trabalho, realizado no campus Duse Rüegger Ometto, da Fundação Hermínio Ometto em Araras, SP, teve a finalidade de correlacionar a distribuição da granulometria do solo e sua permeabilidade com a capacidade de evapotranspiração da área vegetada, pelo método de Biudes (2006) e sua relação com o microclima no local. 


\section{MATERIAL E MÉTODOS OU METODOLOGIA}

\section{1 Área de Estudo}

A área de estudo está localizada no Município de Araras, Estado de São PauIo, na Av. Dr. Maximiliano Baruto, 500 - Jardim Universitário, com localização geográfica $22^{\circ} 22^{\prime} 28.924^{\prime \prime} S$ de latitude e 47022'11.295" W de longitude. As amostras foram coletadas em 5 pontos estratégicos, de modo que fosse representativo de toda a área do Campus Duse Rüegger Ometto- FHOIUniararas (Figura 1).

Figura 2 - Visão geral da área do Campus Duse Rüegger Ometto, FHOIUniararas e os locais dos pontos de coleta do solo para determinação de sua granulometria

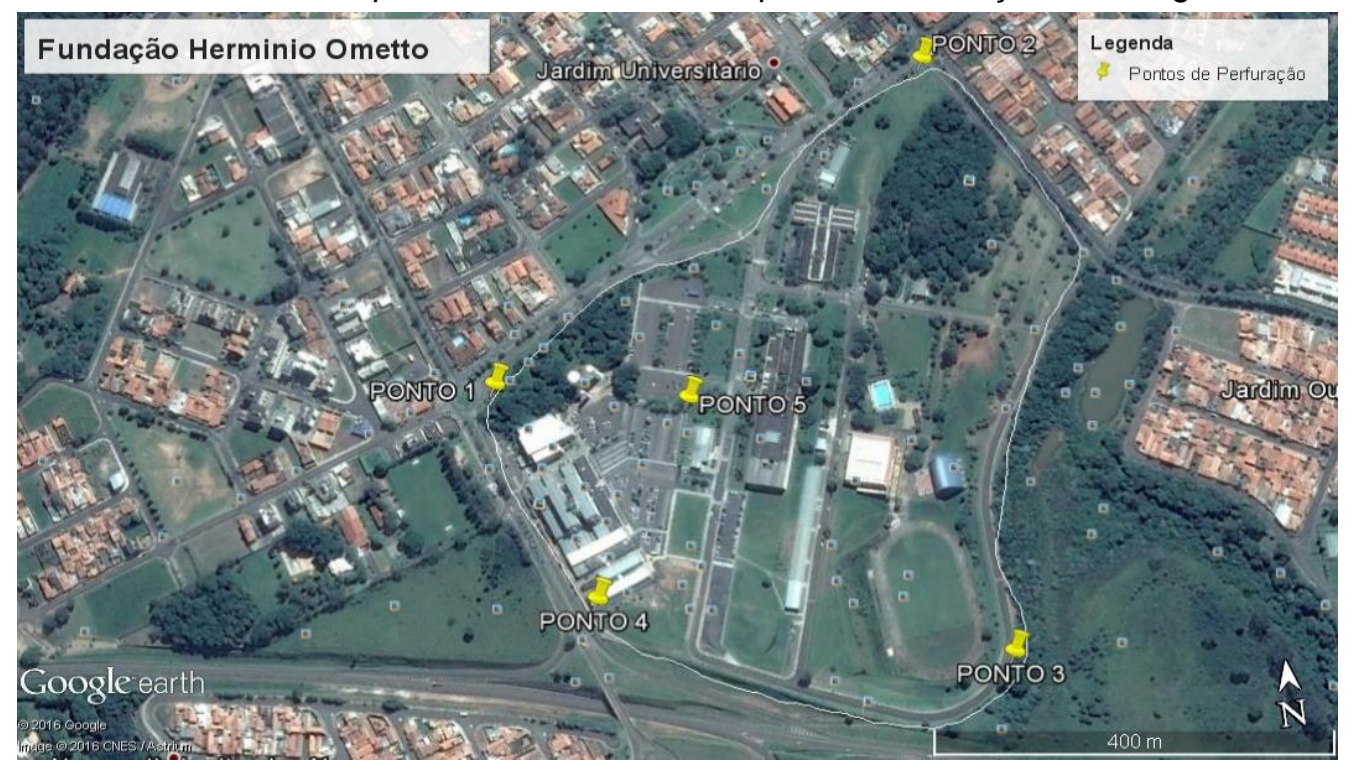

Fonte: https://www.google.com/int/pt-BR/earth/download/ge/agree.html (2016)

O perfil geológico da cidade onde foi realizado o presente estudo é a formação Irati e Corumbataí. Segundo Zaine (1994) a Formação Irati constitui a unidade litoestratigráfica da Bacia do Paraná com maior homogeneidade e identidade em toda a sua extensão areal correspondendo a uma fase de grande estabilidade tectônica da bacia. Gama Jr. (1982 apud ZAINE, 1994) cita também que "define-se como Formação Corumbataí, a unidade superior do Grupo Passa Dois no Estado de São Paulo, com área de ocorrência bastante extensa no vale do Rio Corumbataí" (Figura 3). 


\subsection{Materiais Utilizados}

Os seguintes materiais foram necessários para determinação da granulometria do solo: cavadeira articulada, balança digital (Marte, modelo AD5002), peneirador I-1016 (Contenco), termômetro (minipa MT-350), estufa de secagem e esterilização (Fanem, modelo 320-SE circulação mecânica), peneiras de malhas 16, 30, 50, 80, 100 e 200, embalagens herméticas, trena, vasilhas, amostras de solo e EPI's como óculos de proteção, máscara e luva de couro.

\subsection{Metodologia}

Para coleta das amostras do solo foram utilizados uma cavadeira articulada e um termômetro a laser para medição da temperatura da amostragem no exato momento da coleta. Em cada ponto coletou-se três amostras com as profundidades variando entre $0-20 \mathrm{~cm}, 20-40 \mathrm{~cm}$ e $40-60 \mathrm{~cm}$. No campo foi realizado o teste táctil visual para uma classificação inicial do solo. A armazenagem das amostras foi feita em embalagens herméticas, para preservação da umidade natural, e etiquetadas.

No laboratório todas as amostras foram pesadas separadamente em uma vasilha padrão e posteriormente secadas em estufa à temperatura de $110{ }^{\circ} \mathrm{C}$. Feito isso, as amostras foram pesadas novamente para determinação da diferença de umidade do solo.

As amostras secas puderam então ser colocadas no peneirador durante 10 minutos para separação dos grãos nas respectivas malhas conforme a composição amostral.

Ao final do peneiramento as amostras foram pesadas afim de determinar a porcentagem granulométrica retida em cada malha (Figura 4). 
Figura 3 - Coluna Estratigráfica da Bacia do Paraná no estado de São Paulo

\begin{tabular}{|c|c|c|c|c|c|c|c|}
\hline \multicolumn{2}{|c|}{ COLUNA } & \multicolumn{2}{|c|}{ ESTRATIGRÁFICA D } & A BACIA & DO PARANÁ & \multicolumn{2}{|c|}{ NA REGIÃO DE RIO CLARO/LIMEIRA/PIRACICABA (SP) } \\
\hline$\stackrel{\$}{\mathbf{w}}$ & PERÍODOS & 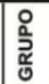 & FORMAÇÃO & LITOLOGIA & \begin{tabular}{|c|}
$\begin{array}{c}\text { Espes. } \\
\text { Appox } \\
\text { (motros }\end{array}$ \\
\end{tabular} & DESCRIÇÃO SUCINTA & $\begin{array}{l}\text { AMBIENTE DE } \\
\text { DEPOSIÇÃO }\end{array}$ \\
\hline \multirow{2}{*}{ 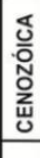 } & QUATERNÁRIO & & RIO CLARO & & 30 & $\begin{array}{l}\text { ARENITOS POUCO CONSOLIDADOS COM LENTES DE } \\
\text { ARGILAS E NIVEIS CONGLOMERATICOS NA BASE }\end{array}$ & $\begin{array}{l}\text { CONTINENTAL: PLANICIE ALUVIAL } \\
\text { E LACUSTRE. COLUVIÖES }\end{array}$ \\
\hline & TERCIÁRIO & & ITAQUERI & & 100 & $\begin{array}{l}\text { ARENITOS CONGLOMERÁTICOS E ARENITOS } \\
\text { SILICIFICADOS / FERRICRETES }\end{array}$ & $\begin{array}{l}\text { CONTINENTAL: LEQUES ALUVIAIS } \\
\text { FLUVIAL E LACUSTRE }\end{array}$ \\
\hline \multirow{4}{*}{ 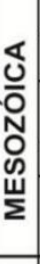 } & CRETÁCEO & \multirow{4}{*}{ 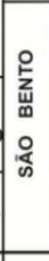 } & SERRA GERAL & & 100 & $\begin{array}{c}\text { DERRAMES DE BASALTOS COM LENTES } \\
\text { DE ARENITO NA BASE. DIQUES E SOLEIRAS } \\
\text { DE DIABÁSIO } \\
\end{array}$ & MAGMATISMO FISSURAL \\
\hline & JURÁSSICO & & ВОTUCATU & & 100 & $\begin{array}{l}\text { ARENITOS BEM SELECIONADOS COM GRÃOS BEM } \\
\text { ARREDONDADOS E BEM ESFERICOS, POUCA ARGILA }\end{array}$ & CONTINENTAL: DESÉRTICO \\
\hline & & & & & & \begin{tabular}{|r} 
Aqüiffero \\
Guarani
\end{tabular} & \\
\hline & TRIÁSSICO & & PIRAMBÓIA & & 150 & $\begin{array}{l}\text { ARENITOS COM GRĀOS ARREDONDADOS E } \\
\text { ESFÉRICOS. DIVERSOS NIVEIS DE LAMITOS }\end{array}$ & CONTINENTAL: FLUVIAL E DESÉRTICO \\
\hline \multirow{5}{*}{ 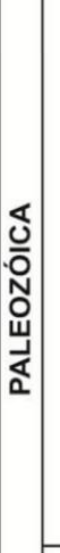 } & \multirow{5}{*}{ 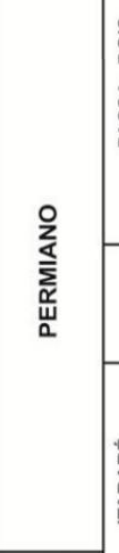 } & \multirow{4}{*}{ 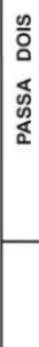 } & CORUMBATAÍ & & 100 & $\begin{array}{l}\text { SILTITOS CONTENDO LENTES DE ARENITOS FINOS } \\
\text { ARGILITOS, SILTITOS, ARENITOS FINOS, } \\
\text { NIVEIS DE CALCARIOS DOLOMITICOS E COQUINAS }\end{array}$ & $\begin{array}{l}\text { CONTINENTAL: LACUSTRE } \\
\text { TRANSICIONAL: PLANICIE DE MARE }\end{array}$ \\
\hline & & & & & & $\begin{array}{c}\text { (Argilitos = matéria-prima para a indústria } \\
\text { cerâmica da regiäo de Rio Claro) }\end{array}$ & \\
\hline & & & IRATI $\frac{\text { Mb. Assistôncia }}{\text { Mb. Taquaral }}$ & & 40 & $\begin{array}{c}\text { FOLHELHOS, SILTITOS, } \\
\text { FOLHELHOS } \\
\text { PIROBETUMINOSOS, CALCARIOS DOLOMITICOS }\end{array}$ & $\begin{array}{l}\text { TRANSICIONAL: LAGUNA } \\
\text { MARINHO RASO: PLATAFORMA } \\
\end{array}$ \\
\hline & & & TATUI & & 50 & SILTITOS E SILTITOS ARENOSOS & $\begin{array}{l}\text { TRANIICIONAL: PLANICIE COSTEIRA } \\
\text { MARINHO RASO: PLATAFORMA }\end{array}$ \\
\hline & & 崖 & $\begin{array}{l}\text { Grupo ITARARÉ } \\
\text { (indiviso no } \\
\text { Estado de Sâo Paulo) }\end{array}$ & $\frac{5-1}{2}$ & 㝵 900 & $\begin{array}{l}\text { ARENITOS, SILTITOS, VARVITOS E } \\
\text { DIAMICTITOS( ALGUNS VERDADEIROS TILITOS) }\end{array}$ & $\begin{array}{l}\text { CONTINENTAL (GLACIALL): } \\
\text { ALUVIAL. LEQUES E FLUVIAL; } \\
\text { LACUSTRE } \\
\text { TRANSICIONAL: DELTAS } \\
\text { MARINHO (GLÁCIO-MARINHO): } \\
\text { PLATAFORMAL }\end{array}$ \\
\hline \multicolumn{2}{|c|}{ Pré-Cambriano } & & EMBASAMENTO & & & $\begin{array}{l}\text { GRANITOS, MIGMATITOS, GNAISSES, XISTOS, } \\
\text { QUARTZITOS }\end{array}$ & \\
\hline
\end{tabular}

Fonte: Perinotto e Zaine (2008)

Figura 4 - Peneiras utilizadas para segregação do solo, por tamanho, em cinco pontos amostrais no Campus Duse Rüegger Ometto - FHOIUniararas, Araras - SP

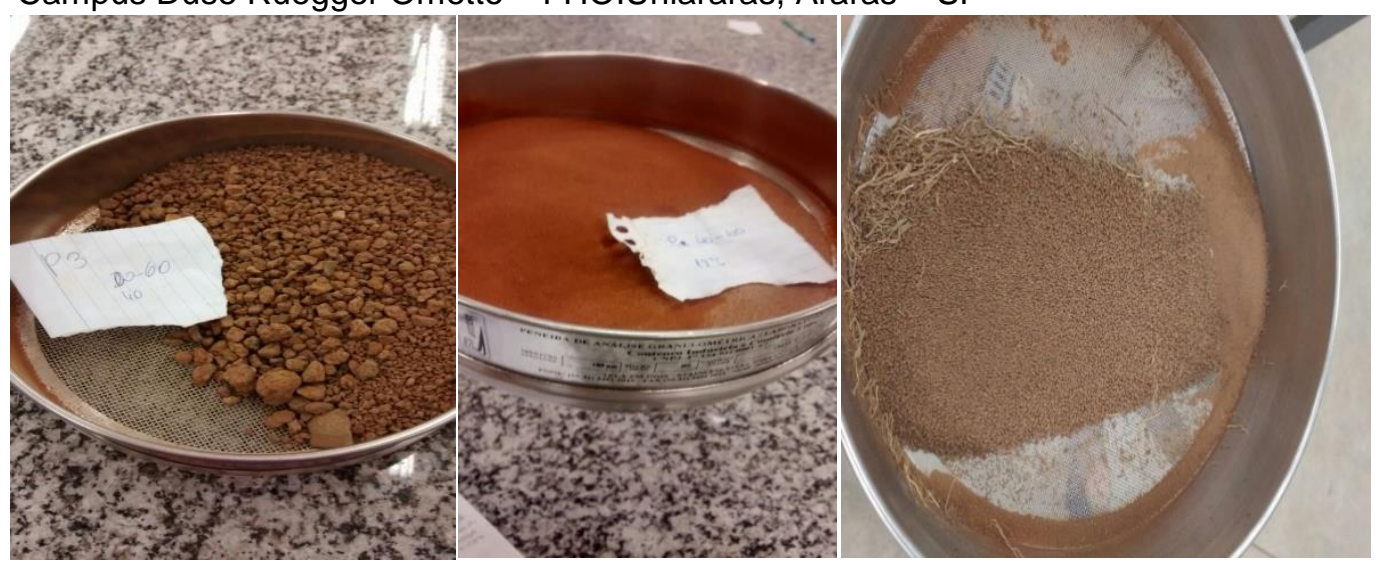

Fonte: Elaborado pelos autores (2016)

Como referência para a classificação do solo, foi usado como base a ABNT NBR 6502, Set. 1995: Areia grossa:0,6mm à 2,0mm; areia média: 0,2 a 0,6mm; 
Areia fina: 0,06 a 0,2mm; Silte: 0,002 a 0,06mm; Argila: partículas menores que $0,002 \mathrm{~mm}$.

Solos arenosos apresentam porosidade média entre 35\% e taxa de permeabilidade em torno de $1 \%$.

Para a determinação da evapotranspiração no Campus Duse Ruegger Ometto, as áreas foram obtidas por duas metodologias, a primeira feita in loco com trenas de 30 metros, e a segunda, delimitadas por satélites e calculadas com o software AutoCad.

As estimativas da transpiração foram desenvolvidas através de Biudes (2006) conforme a equação (1).

$$
T=A F \cdot \frac{s \cdot R n_{f}+900 \cdot \rho \cdot c p \cdot \frac{D e}{r a}}{\lambda\left[s+\gamma \cdot\left(2+\frac{r f}{r a}\right)\right]}
$$

onde $T$ é a transpiração (kg.árvore-1 $\left.30 \mathrm{~min}^{-1}\right), A F$ a área foliar da árvore $\left(\mathrm{m}^{2}\right)$, $s$ a tangente a curva de saturação $\left(\mathrm{kPa} .{ }^{\circ} \mathrm{C}^{-1}\right), R n_{f}$ a energia radiante absorvida pela planta (MJ.m ${ }^{-1}$ de folha.30 $\left.\mathrm{min}^{-1}\right), \rho$ a densidade de ar seco $\left(1,225 \mathrm{~kg} \cdot \mathrm{m}^{-3}\right), c p$ o calor específico do ar seco $\left(1,01 \mathrm{MJ} \mathrm{g}^{-1} .^{\circ} \mathrm{C}^{-1}\right)$, De o déficit de pressão de vapor do ar $(\mathrm{kPa})$, ra a resistência aerodinâmica $\left(\mathrm{s}^{\mathrm{m}} \mathrm{m}^{-1}\right), \lambda$ o calor latente de vaporização da água

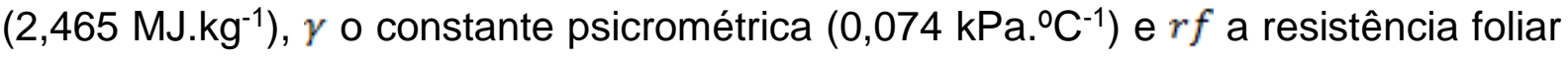
à difusão de vapor $\left(\mathrm{s} \cdot \mathrm{m}^{-1}\right)$.

AF é a área foliar média $\left(\mathrm{m}^{2}\right)$. As áreas estudadas dentro do campus são fruto de reflorestamento ocorrido logo a inauguração da universidade por volta de 1980 , caracterizando assim espaço vegetativo denso e de alturas que giram entorno de 2 a 5 metros. De acordo com consulta aos registros da instituição, as áreas vegetadas são compostas principalmente por espécies nativas, tais como o faveiro (Peltophorum dubium), a peroba (Aspidosperma polyneuron), o carvalho (Quercus sp), o guarantã (Esenbeckia leiocarpa), o ipê (Tabebuia sp), a quaresmeira (Tibouchina sp), a goiabeira (Psidium guajava) e a pitangueira (Eugenia uniflora) plantadas em distribuição uniforme pelo terreno.

Foram coletadas duas folhas de cada em árvores diferentes em cada espécie resultando um total de 16 amostras. Para o cálculo da área foliar individual foi utilizada uma simplificação considerando que cada folha é composta de vários retângulos da base (próxima ao cabo, Figura 5) até a ponta com $01 \mathrm{~cm}$ de altura, com a área de cada retângulo projetado dentro da folha dada pela equação (2). 


$$
A F^{\prime}=B \cdot H
$$

Figura 5 - Exemplar de folha com a demarcação de altura $(H)$ igual a $0,01 \mathrm{~m}$

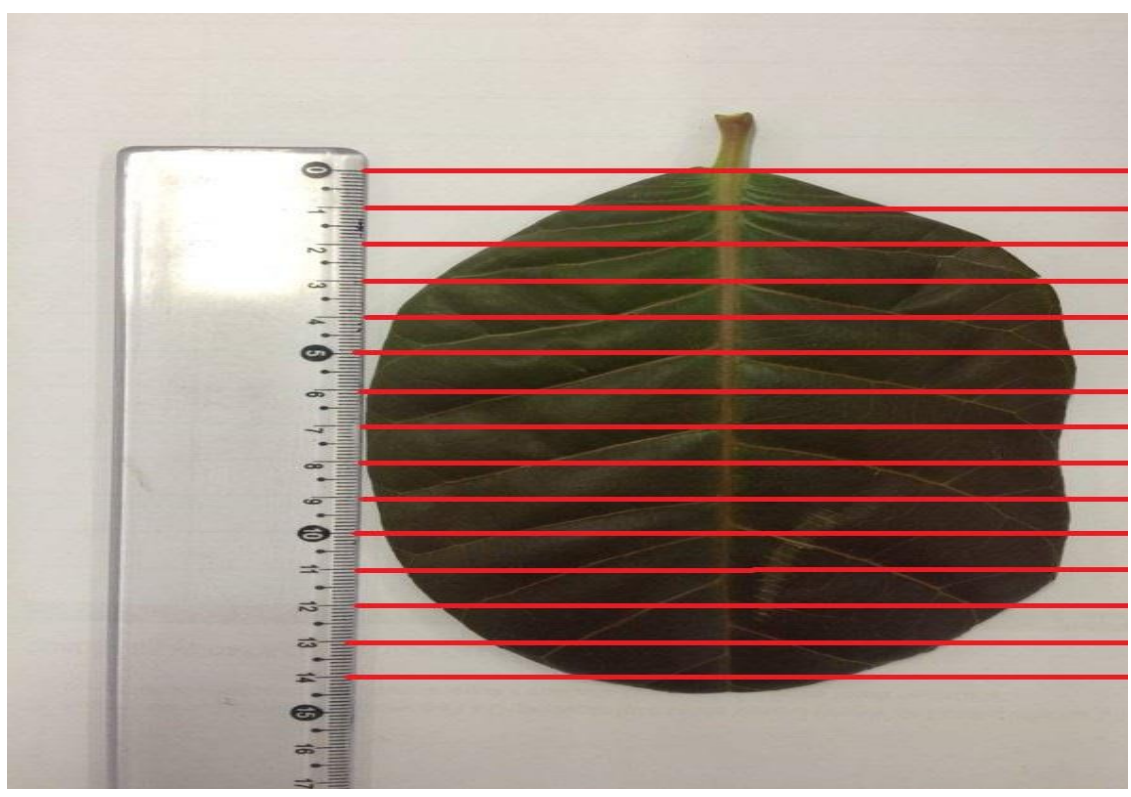

Fonte: Elaborado pelos autores (2017).

onde $B$ é a medida da linha horizontal medida a cada 01 centímetro e dada em metros $(\mathrm{m})$ enquanto $H$ é a altura das folhas coletadas como amostras $(\mathrm{m})$ mas de valor constante e igual a 0,01 m devido a simplificação que possibilitou o cálculo.Obtida a área de cada retângulo projetado dentro da folha, a área foliar total individual foi obtida através da soma dos valores de $A F$ 'área resultando na área foliar média $A F$.

A tangente à curva de pressão de saturação de vapor d'água foi determinada pela equação (3) e $e_{s}$ a pressão de vapor saturado obtido pela fórmula de Tetens (1930) equação (4).

$$
\begin{aligned}
& s=\frac{4098}{(t+237,3)^{2}} \cdot e_{s^{*}}(t) \\
& e_{s}=0,6108 \cdot \exp ^{\frac{17,8, t}{287_{4} 8+t}}
\end{aligned}
$$

onde $t$ é a temperatura de bulbo seco relativo à média dos últimos 30 anos $(\mathrm{em} \stackrel{\circ}{\mathrm{C}})$.

O déficit de pressão de vapor do ar (De) obtido pela equação (5). 


$$
D e=e_{s}-e_{a}
$$

onde $e_{a}$ a pressão de vapor em função da umidade do ar obtida pela Lei de Dalton, equação (6).

$$
e_{a}=P_{H 2 O}
$$

A energia radiante absorvida pela planta $\left(R n_{f}\right)$ por unidade de área foliar (W.m-2 de folha) proposto por Biudes (2006) (Figura 6).

Figura 6 - Valores obtidos para a energia radiante absorvida pela planta $\left(R n_{f}\right)$ por unidade de área foliar, em plantas irrigadas ou não

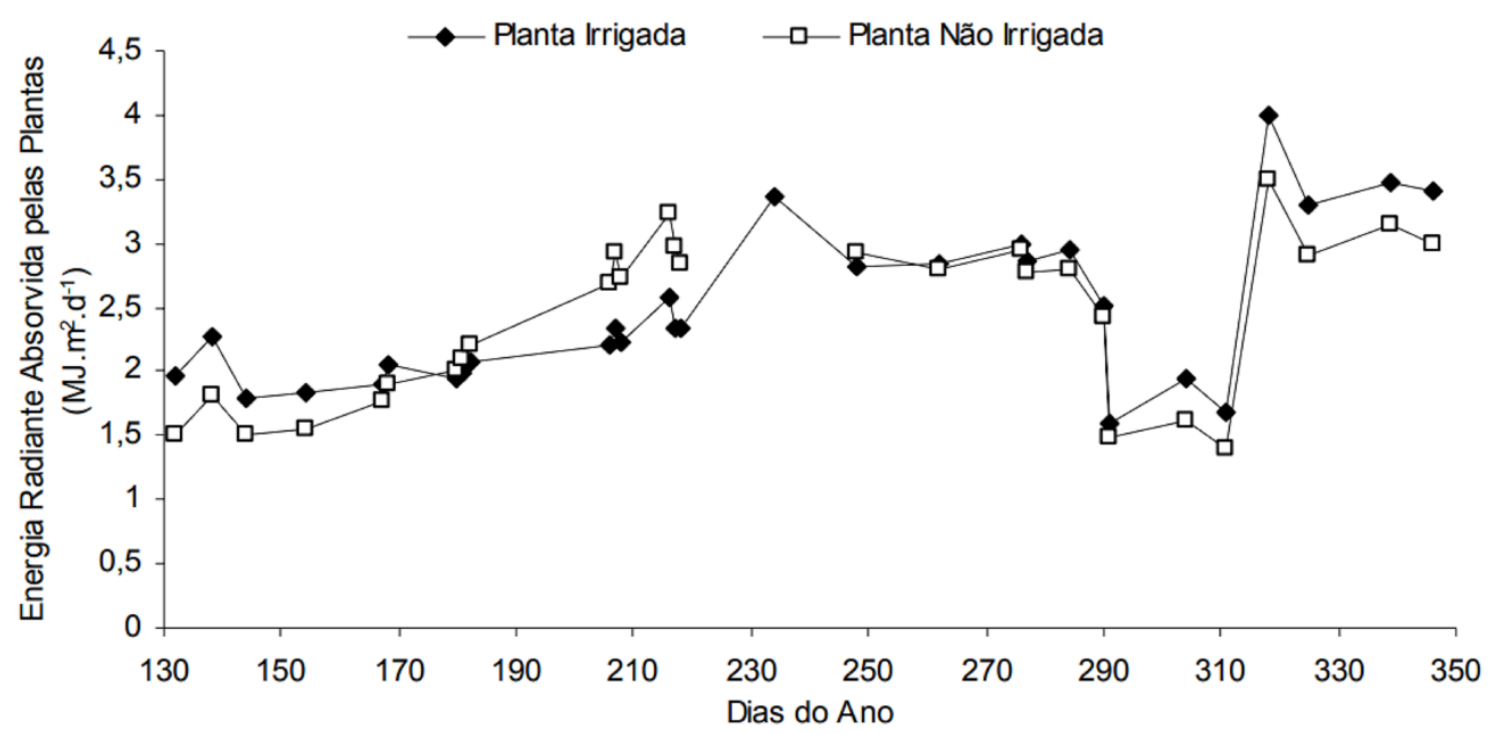

Fonte: Biudes (2006)

Por meio da pesquisa de Zhang et al. (1997) a resistência aerodinâmica das plantas foi obtida de acordo com a equação (7).

$$
r_{a}=58 \cdot p^{0,56} \cdot\left(\frac{\delta}{u}\right)^{0,5}
$$

onde $r_{\alpha}$ a resistência aerodinâmica $\left(\mathrm{s} . \mathrm{m}^{-1}\right), p$ a razão entre a área projetada $\left(A_{\text {proj }}\right)$ equação (8) e silhueta $(S)$ equação (9) do volume da copa de cada árvore na direção predominante do vento (adimensional), $\delta$ é uma dimensão característica das folhas $(\mathrm{m})$ e considerada igual à raiz quadrada da área média das folhas da árvore 
$(A F)$ equação (2), e $u$ a velocidade do vento $\left(\mathrm{m}^{\mathrm{s}} \mathrm{s}^{-1}\right)$ obtido pelo mapa de isopletas da NBR 6123.

$$
\begin{aligned}
& A_{\text {proj }}=\pi \cdot\left(\frac{D_{1}+D_{2}}{4}\right)^{2} \\
& S=\pi \cdot\left[\frac{\left(H_{1}-H_{2}\right)+\left(\frac{D_{1}+D_{2}}{2}\right)}{2}\right]^{2}
\end{aligned}
$$

Onde $D_{1}$ e $D_{2}$ são os diâmetros máximos e mínimos horizontais $(\mathrm{m})$ da copa e $H_{1}$ a altura do dossel desde o solo $(\mathrm{m})$ e $H_{2}$ a distância entre o solo e a parte inferior da copa $(m)$.

A resistência foliar à difusão de vapor $(r f)$ foi estimada através da média das ordenadas da Figura 7 para plantas não irrigadas proposto por Biudes (2006).

Figura 7 - Valores obtidos para a resistência foliar à difusão de vapor $(r f)$, em plantas irrigadas ou não

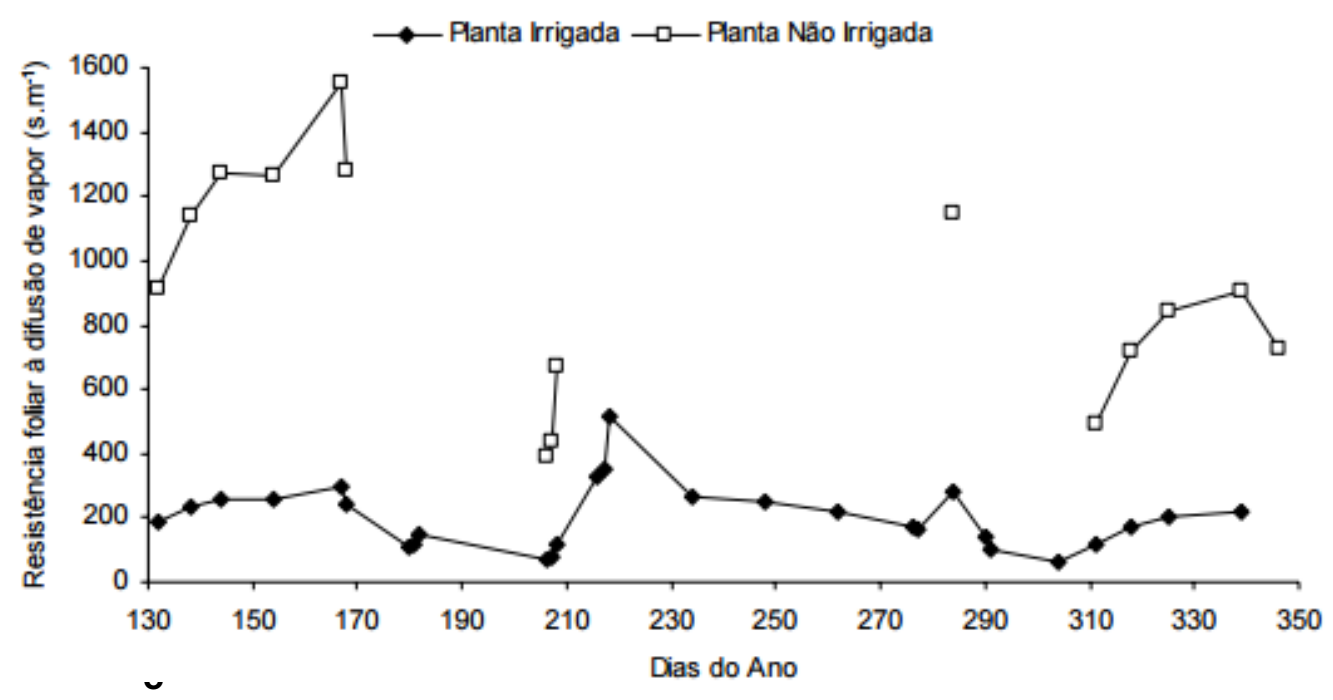

Fonte: Biudes (2006)

\section{RESULTADOS E DISCUSSÃO}

As análises dos cinco (5) pontos amostrais de solo quanto a sua granulometria, no Campus Duse Rüegger Ometto, estão na Tabela 1.

Dos dados analisados pode-se classificar o solo, de uma maneira geral, para as cinco amostras retiradas do Campus Duse Rüegger Ometto - FHOIUniararas, 
pelo método táctil como sendo correspondente a um solo arenoso, pouco argiloso, de constituição fina a média.

O solo, por meio das análises obtidas, está constituído por predominância de areia fina e média com pouca significância de argila. Isso indica um elevado índice de vazios e alta taxa efetiva de porosidade, o que favorece a percolação da água no solo.

Foi observado que em algumas peneiras o solo ficou retido, o que indica que o material não estava completamente seco, portanto o tempo de secagem das amostras não foi suficiente. O solo foi coletado como estava sem se retirar os materiais estranhos, assim as primeiras camadas de 0-20 cm continham além de solo outros materiais, como grama, cimento, pregos, raízes.

Percebeu-se que houve diferença entre o peso da amostra seca e o peso somado das malhas após a peneiração, essa diferença se deve a perdas de material durante a manipulação das amostras.

Referente a umidade do solo, o Ponto 3 apresentou maior taxa de umidade devido sua localização ser próxima a um curso de água. A taxa média de umidade variou de $2,6 \%$ a $8,4 \%$.

Bufon et al. (2016) analisando o terreno do Campus da FHOIUniararas, por meio de curvas de nível, constataram que a área apresentou um desnível de 44 metros em relação ao divisor de água (ponto 1, com 674 m) com o fundo de vale (ponto 3, $640 \mathrm{~m})$, onde se encontra o Córrego do Andrezinho (630 m). A distância entre o ponto 1 com o córrego é cerca de 700 metros.

O ponto 3 se localiza o mais próximo do córrego, o que justifica a maior taxa de umidade encontrada entre as amostras coletadas. 
Tabela 1 - Granulometria de cinco pontos de amostras de solo do Campus Duse Rüegger Ometto FHOIUniararas segregados por diferentes tamanhos de peneira de solo

\begin{tabular}{|c|c|c|c|c|c|c|c|c|c|}
\hline \multicolumn{10}{|c|}{ Malhas } \\
\hline Pontos & \multicolumn{2}{|c|}{$\begin{array}{l}\text { Profundidade } \\
\text { Fundos } \\
\text { do solo (cm) }\end{array}$} & 30 & 50 & 80 & 100 & 200 & & \\
\hline \multirow{5}{*}{1} & $0-20$ & 21,69 & 71,99 & 231,71 & 286,49 & 121,49 & 175,59 & 55,92 & \multirow{5}{*}{ e } \\
\hline & $20-40$ & 200,54 & 68,23 & 191,99 & 328,23 & 115,23 & 139,57 & 38,38 & \\
\hline & $40-60$ & 20,79 & 46,46 & 341,54 & 94,40 & 10,25 & 11,97 & 3,17 & \\
\hline & Somatória & $\begin{array}{l}243,02 \\
\text { Areia }\end{array}$ & 186,68 & 765,24 & 709,12 & 246,99 & 327,13 & $\begin{array}{l}97,47 \\
\text { Argila }\end{array}$ & \\
\hline & Classificação & $\begin{array}{l}\text { Grossa } \\
9,44\end{array}$ & \multicolumn{3}{|c|}{ Areia Média 36,96 } & \multicolumn{2}{|c|}{ Areia fina 49,20} & silte 3,78 & \\
\hline \multirow{6}{*}{2} & $0-20 \mathrm{~cm}$ & 28,18 & 53,60 & 107,57 & 272,27 & 134,06 & 178,29 & 29,83 & \multirow{6}{*}{ e } \\
\hline & $20-40$ & 3,84 & 122,98 & 109,61 & 546,57 & 144,62 & 28,19 & 24,65 & \\
\hline & $40-60$ & 81,24 & 59,43 & 164,61 & 312,7 & 73,51 & 60,25 & 11,57 & \\
\hline & Somatória & 113,26 & 236,01 & 381,79 & 1131,54 & 352,19 & 266,73 & 66,05 & \\
\hline & & Areia & \multirow{2}{*}{\multicolumn{3}{|c|}{ Areia Média 24,25 }} & \multirow{2}{*}{\multicolumn{2}{|c|}{ Areia fina 68,71}} & Argila & \\
\hline & Classificação & $\begin{array}{l}\text { Grossa } \\
4,45\end{array}$ & & & & & & silte 2,59 & \\
\hline \multirow{6}{*}{3} & $0-20$ & 68,88 & 81,08 & 359,34 & 355,81 & 47,26 & 61,70 & 15,85 & \multirow{6}{*}{ e } \\
\hline & $20-40$ & 201,43 & 68,09 & 252,41 & 368,57 & 122,57 & 70,31 & 10,17 & \\
\hline & $40-60$ & 564,92 & 103,79 & 210,40 & 209,90 & 87,47 & 93,84 & 13,98 & \\
\hline & Somatória & 844,23 & 252,96 & 822,15 & 934,28 & 257,30 & 225,85 & 39,99 & \\
\hline & & Areia & \multirow{2}{*}{\multicolumn{3}{|c|}{ Areia Média 31,84 }} & & & Argila & \\
\hline & Classificação & $\begin{array}{l}\text { Grossa } \\
25,00\end{array}$ & & & & \multicolumn{2}{|c|}{ Areia fina 41,98} & silte 1,18 & \\
\hline \multirow{6}{*}{4} & $0-20$ & 141,76 & 85,52 & 543,62 & 157,94 & 25,90 & 26,70 & 5,01 & \\
\hline & $20-40$ & 142,15 & 119,46 & 524,67 & 150,75 & 36,98 & 38,07 & 7,16 & \\
\hline & $40-60$ & 136,86 & 286,86 & 0,54 & 507,47 & 134,53 & 99,25 & 16,97 & \\
\hline & Somatória & 420,77 & 490,84 & 1068,83 & 816,16 & 197,41 & 164,02 & 29,14 & \\
\hline & Classificação & Areia & \multirow{2}{*}{\multicolumn{3}{|c|}{ Areia Média 48,94 }} & \multirow{2}{*}{\multicolumn{2}{|c|}{ Areia fina 36,95}} & Argila & e \\
\hline & & $\begin{array}{l}\text { Grossa } \\
13.20\end{array}$ & & & & & & silte 0,91 & \\
\hline \multirow{6}{*}{5} & $0-20$ & 70,43 & 57,20 & 245,76 & 61,98 & 23,58 & 31,03 & 5,35 & \\
\hline & $20-40$ & 71,29 & 39,86 & 103,37 & 202,89 & 91,05 & 108,02 & 30,2 & \\
\hline & $40-60$ & 152,40 & 43,51 & 72,25 & 100,25 & 54,25 & 72,03 & 30,2 & \\
\hline & Somatória & 294,12 & 140,57 & 421,38 & 365,12 & 168,88 & 211,08 & 65,75 & \\
\hline & Classificação & Areia & \multirow{2}{*}{\multicolumn{3}{|c|}{ Areia Média 33,71 }} & \multirow{2}{*}{\multicolumn{2}{|c|}{ Areia fina 44,70}} & Argila & e \\
\hline & & $\begin{array}{l}\text { Grossa } \\
17,64\end{array}$ & & & & & & silte 3,94 & \\
\hline
\end{tabular}

Fonte: Elaborada pelos autores (2017)

Em relação aos valores de evapotranspiração, de acordo com a metodologia adotada, os valores de áreas obtidas foram: $A_{1}=13$ 101,2742 m², $A_{2}=24381,9906$ $\mathrm{m}^{2}$ e $\mathrm{A}_{3}=20244,8036 \mathrm{~m}^{2}$, cuja soma total das três áreas consideradas foi $\sum$ total $=$ $57728,068 \mathrm{~m}^{2}$

Constatou-se que a área de influência de uma árvore é de $6 \mathrm{~m}^{2}$, portanto o número de árvores é dado por: $\frac{\Sigma}{6}=9621$ árvores.

No entanto é levado em conta as árvores posicionadas em canteiros. Obtidas por contagem direta resultando um total de 294 árvores.

$\therefore 9621+294=9915$ árvores. 
Os dados obtidos com o cálculo da área foliar média $(A F)$ realizada permitiu obter valor médio de 0,00709 (Tabela 2). Há diferenças encontras quanto ao registro da área foliar pode ser explicada por se tratar por diferentes espécies arbóreas nativas, cada uma com sua característica foliar, que pode incrementar a evapotranspiração quanto se observa as espécies usadas na recuperação do campus Duse R. Ometto.

\begin{tabular}{cc}
\multicolumn{3}{c}{ Tabela 2 - Área foliar média } \\
\hline AMOSTRA & ÁREA $\left.\mathbf{( M}^{\mathbf{2}}\right)$ \\
\hline $\mathbf{1}$ & 0,03941 \\
$\mathbf{2}$ & 0,00615 \\
$\mathbf{4}$ & 0,00742 \\
$\mathbf{5}$ & 0,00564 \\
$\mathbf{6}$ & 0,01101 \\
$\mathbf{7}$ & 0,00361 \\
$\mathbf{8}$ & 0,00127 \\
$\mathbf{9}$ & 0,00039 \\
$\mathbf{1 0}$ & 0,01188 \\
$\mathbf{1 1}$ & 0,00065 \\
$\mathbf{1 2}$ & 0,00112 \\
$\mathbf{1 3}$ & 0,00034 \\
$\mathbf{1 4}$ & 0,00243 \\
$\mathbf{1 5}$ & 0,00540 \\
$\mathbf{1 6}$ & 0,01219 \\
\hline MÉDIA & 0,00455 \\
\hline
\end{tabular}

Fonte: Elaborada pelos autores (2017).

Cálculo da tangente à curva de pressão de saturação de vapor d'água. A temperatura de bulbo seco que é igual à temperatura de ambiente foi obtida a partir da temperatura média da cidade de Araras nos últimos 30 anos pelo site do Clima Tempo 2017 (Tabela 3).

Constata-se com a variação maior dos índices registrados para as temperaturas máximas e as mínimas foi no mês de agosto com 13 graus centigrados. De acordo com a equação (4) o valor da pressão de vapor saturado $\left(e_{s}\right)$ é igual a 2,683 $\mathrm{KPa}$ e ainda de acordo com a equação (3) o valor da tangente à curva de pressão de saturação de vapor d'água $(s)$ é igual à $3,624 \mathrm{KPa}$ / ํㅡ. 


\begin{tabular}{|c|c|c|c|}
\hline Mês & Mínima & Máxima & Média \\
\hline Janeiro & 20 & 27 & 23,5 \\
\hline Fevereiro & 20 & 28 & 24,0 \\
\hline Março & 19 & 28 & 23,5 \\
\hline Abril & 17 & 26 & 21,5 \\
\hline Maio & 14 & 23 & 18,5 \\
\hline Junho & 14 & 24 & 19,0 \\
\hline Julho & 14 & 24 & 19,0 \\
\hline Agosto & 15 & 28 & 21,5 \\
\hline Setembro & 17 & 29 & 23,0 \\
\hline Outubro & 19 & 30 & 24,5 \\
\hline Novembro & 19 & 29 & 24,0 \\
\hline Dezembro & 20 & 29 & 24,5 \\
\hline Valores médios & 17,3 & 27,1 & 22,2 \\
\hline
\end{tabular}

As estimativas da transpiração foram desenvolvidas pelo método de Biudes (2006) conforme a equação (1) para árvores isoladas e não regadas calculada em:

$$
\begin{aligned}
& T=0,007334 \cdot \frac{3,624 \cdot 2,50+900 \cdot 1,225 \cdot 1,01 \cdot \frac{1,683}{1,95}}{2,465 \cdot\left[3,624+0,074 \cdot\left(2+\frac{908}{1,95}\right)\right]} \\
& T=0,0745 \text { Kg.árvore }{ }^{-1} \cdot 30 \text { minutos }^{-1} \\
& \therefore T=3,5 \text { litros.árvore }{ }^{-1} .24 \text { horas }^{-1}
\end{aligned}
$$

Relacionando a transpiração de uma árvore isolada com o número de árvores da área vegetada em estudo, tem-se:

$$
\begin{aligned}
& T_{\text {total }}=3,5 \text { (litros.árvore }{ }^{-1} .24 \text { horas }^{-1} \text { ) } .9915 \text { (árvores) } \\
& \therefore T_{\text {total }}=34,7 \mathrm{~m}^{3} .24 \text { horas }^{-1}
\end{aligned}
$$

Segundo Biudes (2006), a qualidade das medidas de fluxo de seiva obtidas pelo método do balanço de calor no caule é difícil de ser avaliada em condições de campo. Visto que é impraticável uma medição direta e precisa dos valores de transpiração de árvores isoladas, uma vez que, as condições climáticas interferem de forma intensa nos resultados.

A energia radiante absorvida pela planta $\left(R n_{f}\right)$ foi estimada a partir de média aritmética das ordenadas (Figura 5), segundo Biudes (2006), para plantas não irriga- 
das, com valor de $R n_{f}=2,50$ W.m-2 (Tabela 4).

Para períodos com baixa umidade do ar e maiores velocidades de ventos os estômatos são propícios a liberar mais vapor de água. Já em dias com maior umidade e menores velocidades de ventos, estes são propícios a reter tal vapor diminuindo os índices de transpiração de uma planta.

É importante destacar que acrescentar que em temperaturas superiores a 25 ${ }^{\circ} \mathrm{C}$, a vegetação não contribui significantemente com evapotranspiração, uma vez que seus estômatos vão se fechando à medida que a temperatura se eleva e a velocidade do vento aumenta, evitando a perda de água para o (DIMOUDI e NIKOLOPOULOU, 2003). Considerando-se tal fato relacionado a uma área vegetada extensa, pode-se supor que outros métodos a serem aplicados poderiam ter visão mais robusta na avaliação das informações coletadas.

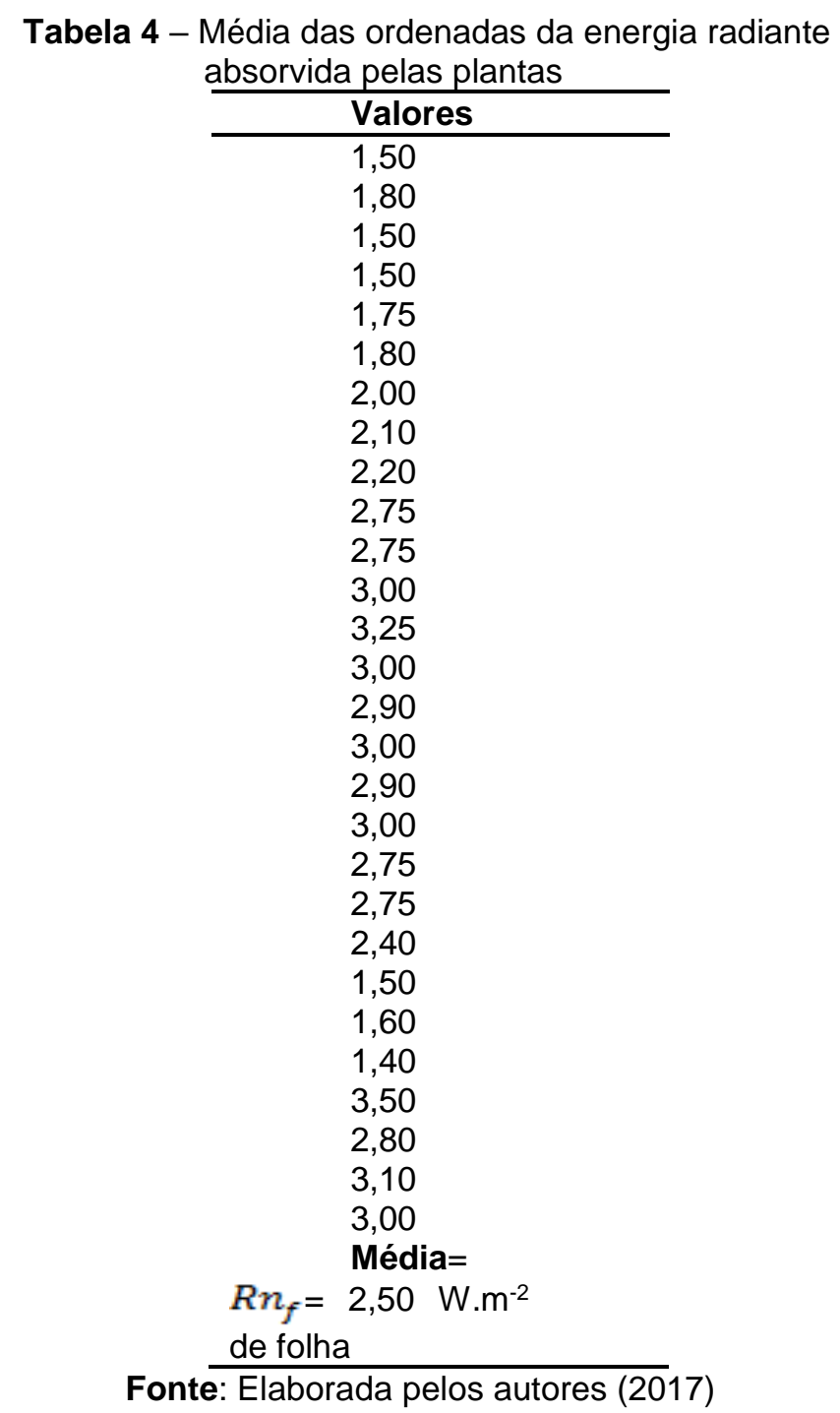


A resistência foliar à difusão de vapor $(r f)$ foi estimada pela média das ordenadas da Figura 7 para plantas não irrigadas proposto por Biudes (2006) (Tabela 5), cuja média obtida da resistência foliar foi de $908 \mathrm{~s} . \mathrm{m}^{-1}$

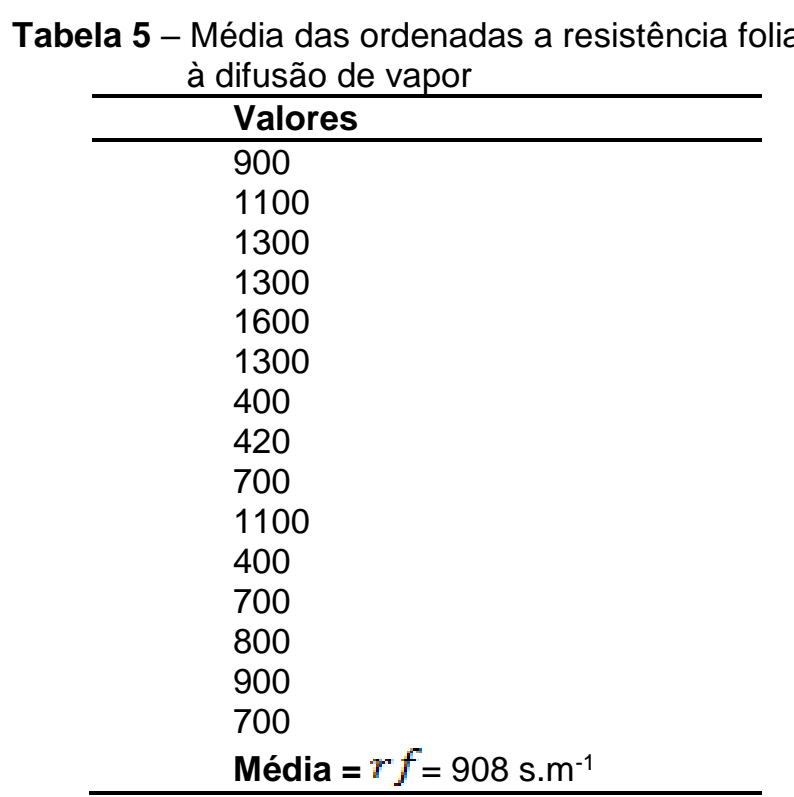

Fonte: Elaborada pelos autores (2017)

O método estipulou um potencial para as condições adotadas e descritas durante o cálculo que procurou ser conservador a fim de evitar discrepâncias exageros. Mesmo assim, o valor obtido de $34,7 \mathrm{~m}^{3} .24$ horas $^{-1}$ representa uma quantidade de água muito grande.

Ressalta-se que o potencial de transpiração de uma área vegetada (árvores, arbustos, forração e trepadeiras) é relevante na melhoria das condições do microclima de uma edificação civil (elementos construídos, como pergolados, espelhos d'água, fontes, entre outros), sendo promissor sua inserção nos ambientes urbanos a fim de melhorar a qualidade do ar e consequente conforto térmico (ABREU, 2008).

Silva, Gonzalez e Silva Filho (2011) afirmam que vegetação em áreas urbanas pode minimizar a aridez do clima urbano pela regulação higrométrica. No entanto, a impermeabilização do solo e a redução da cobertura arbórea, favorecem o escoamento superficial e a intercepção, reduzindo a quantidade de água que infiltra no solo.

\section{CONCLUSÕES}

A análise da granulometria na área do Campus Duse Ruegger Ometto, local 
dessa pesquisa, revelou que houve predominância de areia fina e média com pouca significância de argila, conforme consta na Coluna Estratigráfica da Formação Corumbataí. Isso indica um elevado índice de vazios e alta taxa efetiva de porosidade, o que favorece a percolação da água no solo.

Os valores de transpiração potencial obtidos no presente trabalho sugerem que uma edificação pode se tornar um ambiente aprazível, a partir de uma perceptível queda de temperatura e aumento da umidade do ar oferecida pela vegetação implantada no seu entorno. O valor médio de evapotranspiração obtido, de 34,7 $\mathrm{m}^{3}$.24horas ${ }^{-1}$, corroborando, mais uma vez, a importância da vegetação em áreas urbanas e conforto térmico adequado que propicia e um ambiente menos estressante.

O microclima urbano da região também é passível de intervenção demonstrada a partir de quedas de temperatura locais causadas pelas barreiras de vegetação que envolve o campus.

\section{REFERÊNCIAS}

ASSOCIAÇÃO BRASILEIRA DE NORMAS TÉCNICAS. NBR 6502: Rochas e solos. Rio de Janeiro: Abnt-associação Brasileira de Normas Técnicas, 1995. 18 p.

ABREU, L. V. Avaliação da escala de influência da vegetação no microclima por diferentes espécies arbóreas. 2008. 154 p. Dissertação (Mestrado em Engenharia Civil) - Faculdade de Engenharia Civil, UNICAMP, Campinas - SP.

AMATO-LOURENCO, L. F. et al . Metrópoles, cobertura vegetal, áreas verdes e saúde. Estud. av. São Paulo, v. 30, n. 86, p. 113-130, Apr. 2016.

BIUDES, M. S. Estimativa da transpiração em plantas de mangabeira. 2005. 83 f. Dissertação (Mestrado) - Curso de Física, Departamento de Física, Universidade Federal do Mato Grosso, Cuiabá, 2006. Disponível em:

www.pgfa.ufmt.br/index.php/br/utilidades/dissertacoes/80-marcelo-sacardi.../file.Acesso em: 17 maio 2017.

CLIMA TEMPO (Araras) (Comp.). Climatologia. 2017. Disponível em:

http://www.climatempo.com.br/climatologia/795/araras-s. Acesso em: 10 maio 2017.

FERREIRA, M. M. Caracterização física do solo. In: Lier, Q. J. van (ed.). Física do solo. Viçosa: Sociedade Brasileira de Ciência do Solo, 2010. p.1-27.

LIMA JUNIOR, J. E. de Avaliação da influência de um sistema de fachada viva: o estudo de caso da planta Sphagneticola trilobata em condições de inverno de Curitiba. 2014. 128 p. Dissertação (Mestrado em Engenharia de Construção Civil) - UFPR, Curitiba - PR.

LOSSARDO, L.F.; DAINEZI, P. M. Análise da fragilidade ambiental como subsídio ao planejamento do uso e ocupação do solo no município de Araras-SP. Revista Científica do Cen- 
tro Universitário de Araras “Dr. Edmuldo Ulson" - UNAR, Araras (SP), v. 5, n. 1, p. 3850, 2011.

MACHADO, N.G. et al. Temperatura e umidade relativa do ar na estação seca em diferentes usos do solo no Campus Cuiabá - Bela Vista do IFMT. Revista Eletrônica em Gestão, Educação e Tecnologia Ambiental, Santa Maria - RS, v. 9, n. 9, p. 2018-2025, 2013;

MARTINI, A.; BIONDI, D. Microclima e conforto térmico de um fragmento de floresta urbana em Curitiba, PR. Floresta e Ambiente, Rio de Janeiro, v. 22, n. 2, p. 182-193, 2015.

NOGUEIRA, T. Estômatos. 2006. Disponível em:

http://www.infoescola.com/citologia/estomatos. Acesso em: 17 maio 2016.

PERINOTTO, J. A. J.; ZAINE, M. F. Patrimônios naturais e história geológica da região de Rio Claro-SP. Rio Claro: Arquivo Público e Histórico do Município de Rio Claro, 2008. v. 1.

RIBEIRO, K.D. et al. Propriedades físicas do solo, influenciadas pela distribuição de poros, de seis classes de solos da região de Lavras-MG. Ciênc. agrotec., Lavras, v. 31, n. 4, p. 1167-1175, jul./ago., 2007.

SILVA, I.M.; GONZALEZ, L.R.; SILVA FILHO, D.F. Recursos naturais de conforto térmico: um enfoque urbano. Revista da Sociedade Brasileira de Arborização Urbana, Curitiba, v. 6, n. 4, p. 35-50, 2011.

SANTOS, K. A.; RUFINO, I. A. A.; BARROS FILHO, M. N. M. Impactos da ocupação urbana na permeabilidade do solo: o caso de uma área de urbanização consolidada em Campina Grande - PB. Eng. Sanit. Ambient., Rio de Janeiro, v. 2,2 n. 5, p. 943-952, set./out. 2017.

TOLFFO, F. A. O pagamento por serviços ecossistêmicos como instrumento de gestão ambiental para o espaço urbano. 2015. 289 p. Dissertação (Mestrado em Ambiente, Saúde e Sustentabilidade) - Faculdade de Saúde Pública, Universidade de São Paulo, São Paulo, 2015.

ZAINE, J. E. Geologia da formação Rio Claro na folha Rio Claro (SP). 1994. 98 f. Dissertação (Mestrado em Geologia Regional) - Curso de Geociências, Universidade Estadual Paulista Instituto de Geociências e Ciências Exatas, Rio Claro, 1994.

ZHANG, H. et al. Estimation of transpiration by single trees: comparison of sap flow measurements with a combination equation, Agricultural and Forest Meteorology, v. 87, p. 155169, 1997. 